\title{
Angle-Aware and Tone-Aware Luminosity Analysis for Paper Model Surface
}

\author{
Xiaozhou LI*, Mingming CUI, Xiaomeng HAN, Guangyuan WU, Jingjing LIU
}

\begin{abstract}
Luminosity contributes to the paper model surface perception. It has a significant impact on the perception of colour and details. The main purpose of this paper is to study the reflection luminosity of paper model surface which can be of complex or difficult shape surface. The final perception quality of a product, whether it is plain or 3D or other different shape, depends on the surface luminosity perceived by the receptor, such as eyes or measurement instruments. However, the number of parameters and limits of the paper model surface are enormous. It is a time-consuming work to select every parameter by a trial-and-error procedure. For a paper surface under the fixed lighting environment, the most important factors to decide the performance of perception are commonly viewing angles and surface tone. Therefore, the two related terms, perception angle and surface tone, were chosen to work in the analysis process. The final analysis, based on the initial conditions, enabled to predict the perception of paper model surface and to set the optimal perceived angels and tones. It still proposed the next step to model the perception of paper model surface of different shapes in a relatively short period.
\end{abstract}

Keywords: luminosity; paper model surface; perception angle; reflection spectral measurement; tone value

\section{INTRODUCTION}

Colour analysis of paper model product has been an extremely popular topic in many research areas including colour reproduction, image processing, 3D printing, human skin colour measurement and analysis, etc. For example, luminosity of skin surface, which is similar to 3D paper model surface, is obtained using special under different viewing angles [1]. In addition, translucency is also added to help improving $3 \mathrm{D}$ printing colour quality [2]. The difference is that paper model product is always opaque usually. Kubelka-Munk model is also used to reproduce human skin colour, though it is more common in silk colouration research area [3]. And there are some reports to present colour measurement method for auto surface colour [4]. Reference focused on processes and colour quality evaluation of colour 3D printing [5]. However, with the development of $3 \mathrm{D}$ paper printer, there always lacks a method to predict, measure or evaluate the luminosity of paper product, which is essentially different from the methods used in plain printing colour perception, i.e., 2D colour printing on paper [6]. Luminosity depends on the field of research, i.e., there are different meanings in different area. It means the ratio of luminous flux to radiant flux at the same wavelength in physics while it would mean the ratio of a star radiating energy in stereo directions [7]. It also can be stimulus value in printing colour reproduction. However, in this paper related colour research areas, luminosity refers to the state of colour being "radiant", "brilliant", "lightness", "stimulus" or "luminous", sometimes intensity is always used too [1].

\section{Light Interactions with 3D Paper Model Surface}

In China, paper-based product with different colours is used more and more in culture and creative industries [8]. Because the market is so huge, paper model printed with different colour tones is selected as the main topic in this article. Plain paper is used as the basic substrate applied to demonstrate the culture and creative products, which has advantages including more colourful, easier to produce and lower cost [9]. Additionally, paper-based 3D printing can improve the appearance of the culture and creative products with vivid colours and luminosity. Stereoscopic paper models can be easily produced by several techniques including plain paper printing and packaging techniques, 3D technologies, etc. We took stereoscopic paper models instead of the 3D printed products which make it facilitate to complement. For paper-based colour reproduction, it is mature to predict, measure, reproduce and evaluate the colour perception [6]. While for stereoscopic paper model products, it is still a novel and interesting area for researchers because of complex geometry appearances and viewing conditions. Although there are some researches focused on the colour reproduction using halftone or related screening methods, they are all about the colour reproduction related transparency which has fewer relations with surface luminance perception [10]. It showed that it is easier to use plain surface to simulate the perception of stereoscopic paper model surface. The phenomenon was described easily using the plain paper surface with various angles and tone values together, though there are some differences between stereoscopic paper model and plain paper model. As a result, it is important to evaluate how luminosity changes the appearance of the paper model products. Perception point positions and viewing angles referring to the origin in Cartesian Coordinate System were chosen to measure and analyse the appearance in this paper. The spatial configuration of perceived point and area is shown in Fig. 1 and Fig. 2.

In Fig. 1a, if the position $P$ changes to $P^{\prime}$, the incidence angle $\alpha_{i}$ will change to $\beta_{i}$, which results in reflectance angle $\alpha_{j}$ changing to $\beta_{j}$. In other words, the perceive angle will always change with incidence angle. As a result, the reflect light amount will change and the perception is different for the different $P$ positions. However, for the same position $P$, in Fig. $1 \mathrm{~b}$, the reflectance angle $\theta_{v}$ will always change with the incidence angle $\theta_{i}$. In actual situation, the light source is always set in a fixed position, while the surface is set at a designated angle to the perception position. In Fig.1b, the light source position and the paper surface $P^{\prime}$ are stable to the whole viewing conditions while the perceived angle between the perceptor and $P^{\prime}$ is variable.

For stereoscopic paper model objects, the actual perception conditions always perform as in Fig. 1 and Fig. 2. In Fig. 2, the reflect light, $\mathrm{d} s_{r}$, from the area surface, $\mathrm{d} s$, is always perceived in a solid angle, $\mathrm{d} \Omega_{r}$. For the same 
viewing conditions the appearance will be different for the combination of viewing conditions. It is also described as the directional reflectance characteristics [11-14], which has a famous theory, i.e., BRDF, as follows:

$f_{r}\left(\theta_{i}, \varphi_{i}, \theta_{r}, \varphi_{r}\right) \equiv \frac{\mathrm{d} L_{r}\left(\theta_{i}, \varphi_{i}, \theta_{r}, \varphi_{r}\right)}{\mathrm{d} E_{i}\left(\theta_{i}, \varphi_{i}\right)}$

where, $L_{r}$ is reflected radiance, $\theta$ and $\varphi$ indicate the direction, $i$ indicates quantities associated with incident, $r$ indicates quantities associated with reflected, $E_{i}$ indicates incident irradiance and $\mathrm{d}$ indicates a differential quantity. Because the paper surface is always rough even when printed with inks, the reflected radiance is not scattered into one direction but into different directions. This is shown in Fig. 3.

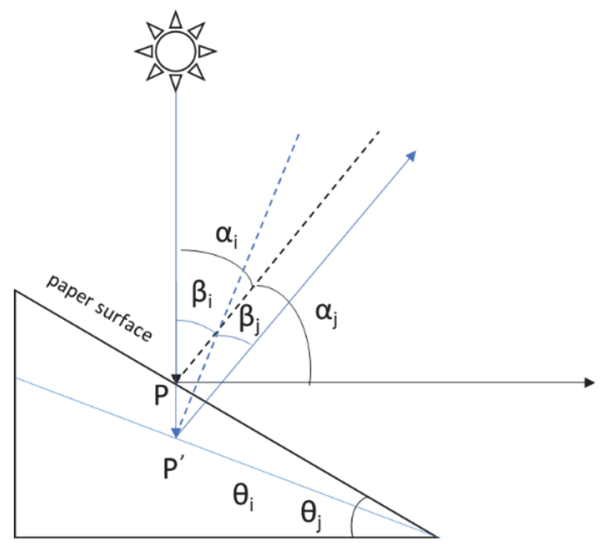

(a) light reflection chart with angle changes

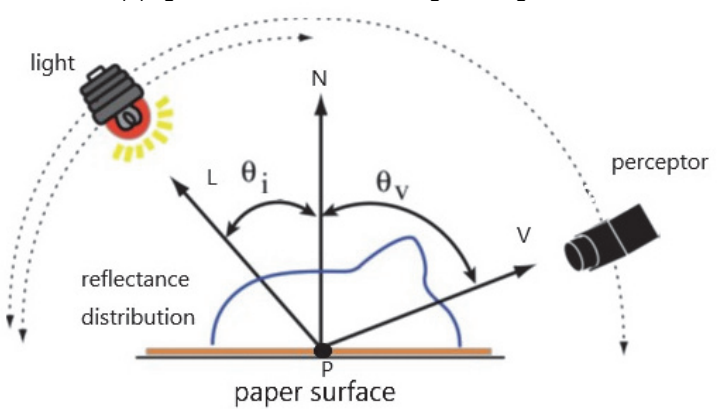

(b) reflection distribution with perceive angles Figure 13D configuration for perception

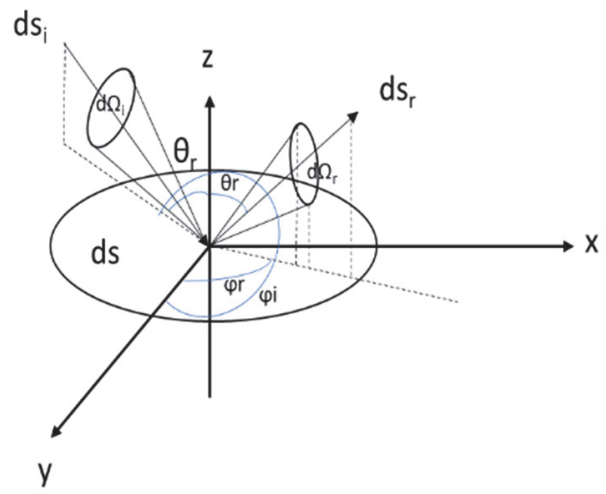

Figure 2 geometry of incident and reflected elementary beams

On the other hand, the perception rule is also suitable for stereoscopic paper model product. Appearance of paper model product surface printed with colour inks changes with the intensity of light reflection, i.e., tone varies. In such case, surface reflection differs according to the tone or ink density. Light reflection on the paper surface with ink often causes absorbance, transmission and multiple reflection between the ink and paper, as shown in Fig. 3.
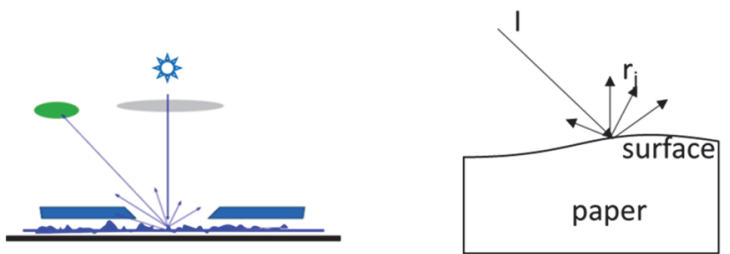

(a)Paper surface with ink dot

(b)light acts on the paper surface

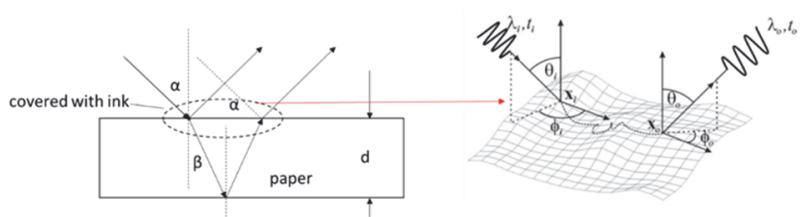

(c) Light reflects, transmits and absorbs on paper surface Figure 3 light directions on paper product surface

In Fig. 3a, the paper surface or paper product surface with ink is always rough. It is covered with ink dots of different sizes. The light with the surface is of diffuse reflection, just like Fig. $3 \mathrm{~b}$ and Fig. 3c. It shows the light perception composition from the paper product surface. With Fig. 1 and Fig. 2, we could find that it is always difficult to evaluate the stereoscopic paper product surface luminosity and colour effectively using traditional method. Consiquently, the colour measure method and prediction method are different from the methods used in 2D or plain printing. And it is also not easy to perceive or predict the luminosity, then colour, of the paper product under a given viewing situation. It is necessary to do the research on the perception of stereoscopic paper product to help solving the problems above.

The luminosity of paper product always changes with variations of viewing angles, as shown in Fig. 1. For the same point, when it transmits to the second position with the perception position stable, the perception would be different as the angle changes. The surface appearance with same tone is different from reflection that is described as luster or specular highlight. Such surface appearance can be best described by terms, e.g., radiant, brilliant, light, stimulus or luminous, of course also in other languages too. Visual evaluation of surface appearance could be perceived by humans with different optical characteristics. Luminosity is now used to show the product luster. And it is also used to represent a total impression of paper model product which gives a better public impression. Various methods of visual evaluation including plain paper product colour with ink in commercial printing, colour appearance attributes using colour appearance model, and 3D computer graphics simulating appearance of paper product surface, are used to evaluate the paper product surface appearance. Although the use of plain paper is popular in colour appearance reproduction or perception, it should be noted that when the luminosity of stereoscopic paper model product is evaluated, various factors would affect the total appearance. These factors include viewing angles, ink 
density or tone, and the illumination environment etc. The factors above hinder the detailed analysis of the visual evaluation and the colour prediction for reproduction in displaying. In order to demonstrate such topic, we would like to take another simple method to describe the "luminance data" distribution which shows the relation between luminance and appearance properties, i.e., complex geometrics of surface and tones. In order to facilitate the description of surface geometrics for paper product, perceived angles related to surface point set positions were used.

For the same surface, it would be perceived with different feeling of luminosity because of the spatial configuration which is shown in Fig. 4a. Surface $A$ and Surface $B$ have same and uniform luminosity if they are perceived in plane under the same view conditions. While we can find that the surface luminosity is not uniform, we also perceive different chroma and luminosity in such configuration. As a result, the stimulus is various even for the same colour tone with different angles referring to the origin in Cartesian Coordinate System as in Fig. 1. For such 3D object like in Fig. 4a, we set different angles for the plain paper to work as the product surface. Of course, there are a couple of differences between the plain paper surface and the real 3D paper product surface, e.g., the roughness is different, Fig. 4b. However, the roughness is not the point discussed in this paper. All surfaces of paper models were produced using the same paper. The surfaces $A$ and $B$ have the same luminosity when they are placed in the same plane. As just discussed above, they are always perceived differently when they are placed in spatial configuration. The key point to describe the difference is to get the relations between the luminosity $A$ and luminosity $B$. As a result, the luminosity or reflection properties to the preceptor must be measured and characterized to help perceive the appearance and prediction of the paper product surface appearance.

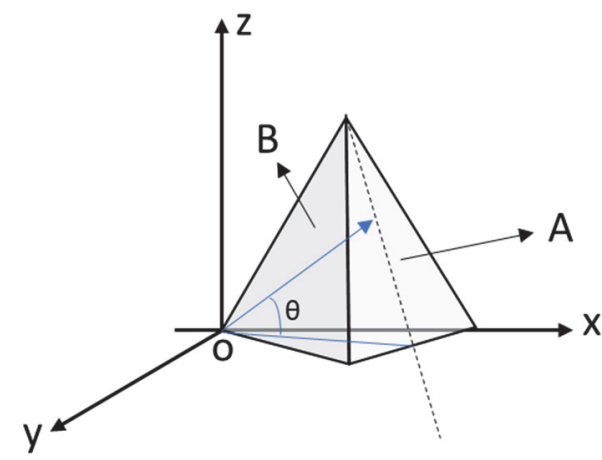

(a) paper model surfaces

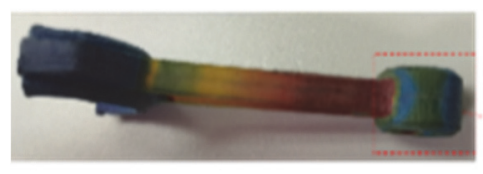

(b) paper model surface roughness

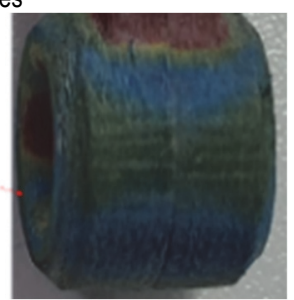

Figure 4 paper model object surfaces and paper surface roughness

Compared to other substrates, it is easier to get more colours on paper surface than on other substrates surface with different methods. Paper product colour can be performed by printing method, coated method or even papermaking method, etc. This article is to identify essential components of the luminosity of 3D paper model product including two processes: luminosity measurement and analysis. A method for perception of surface luminance using a special device and artificial paper model product printed with different tones for the purpose of improving the precision of evaluation is proposed in this paper. The advantage of this method lies in the fact that the paper properties could be obtained using traditional methods, and vivid tones and appearance can be obtained and accessed. Analysis was used to assess luminosity and the relationship of luminosity to the physical amount of reflection or radiation and colour values was analyzed. In addition, the luminosity on paper model surface perceived from different viewing angles was assessed. The luminosity distribution was evaluated on the four primary colour tones on paper product surface. Finally, the results of the luminosity distribution were analyzed in a colour space. The relationship between the luminosity and paper product surface properties, i.e., paper surface position set and tone value, were discussed in this paper.

In the following, the first stage is to perceive the detailed luminosity using different tones and viewing angles. We made paper model samples printed with inks and measured its spectral reflection and radiation characteristic. Furthermore, a special design for this experiment with a group of experimental devices including radiometer, spectrophotometer, colour printer were developed to perceive and evaluate the appearance of the paper model products at different conditions of viewing angle and various tones. Both parameters could affect the luminosity perception. In fact, the illumination may be fixed for the paper model is always perceived with specific lighting configurations. Then the relationship between the luminosity and the surface reflection characteristics were investigated. The third stage is to summarize the luminosity data and analyze the factors in CIE 1931Yxy colour space.

\section{REFLECTION SPECTRAL RADIANT MEASUREMENT OF PAPER SAMPLES WITH DIFFERENT TONES 3.1 Paper Samples Preparation}

Office paper is used as the build material which is very different form expensive materials, e.g., plastic or chemically infused powder. And office paper used in this article is ordinary, affordable and ubiquitous, which is always used in traditional printing. Four primary colour tones, cyan, yellow, magenta and black, were used to print on plain paper from $10 \%$ to $100 \%$ with step of $10 \%$, which is shown in Fig. 5a. And they were set as the surface of paper model. It was maintained at a constant illumination, D65, and had a constant paper surface reflectance. And paper samples with the same tone while different perceived angles are shown in Fig. 5b. The rotation equipment to change the perceived angles like in Fig. 5b, rotating bracket, can also be seen in Fig. 5c. The samples were set on the rotation equipment with angles from 5 degree to 85 degree. The plain paper surface reflection spectral is showed in Fig. 6. It is easy to measure the two-dimentional spectral reflection with spectroradiometer or CS2000. 


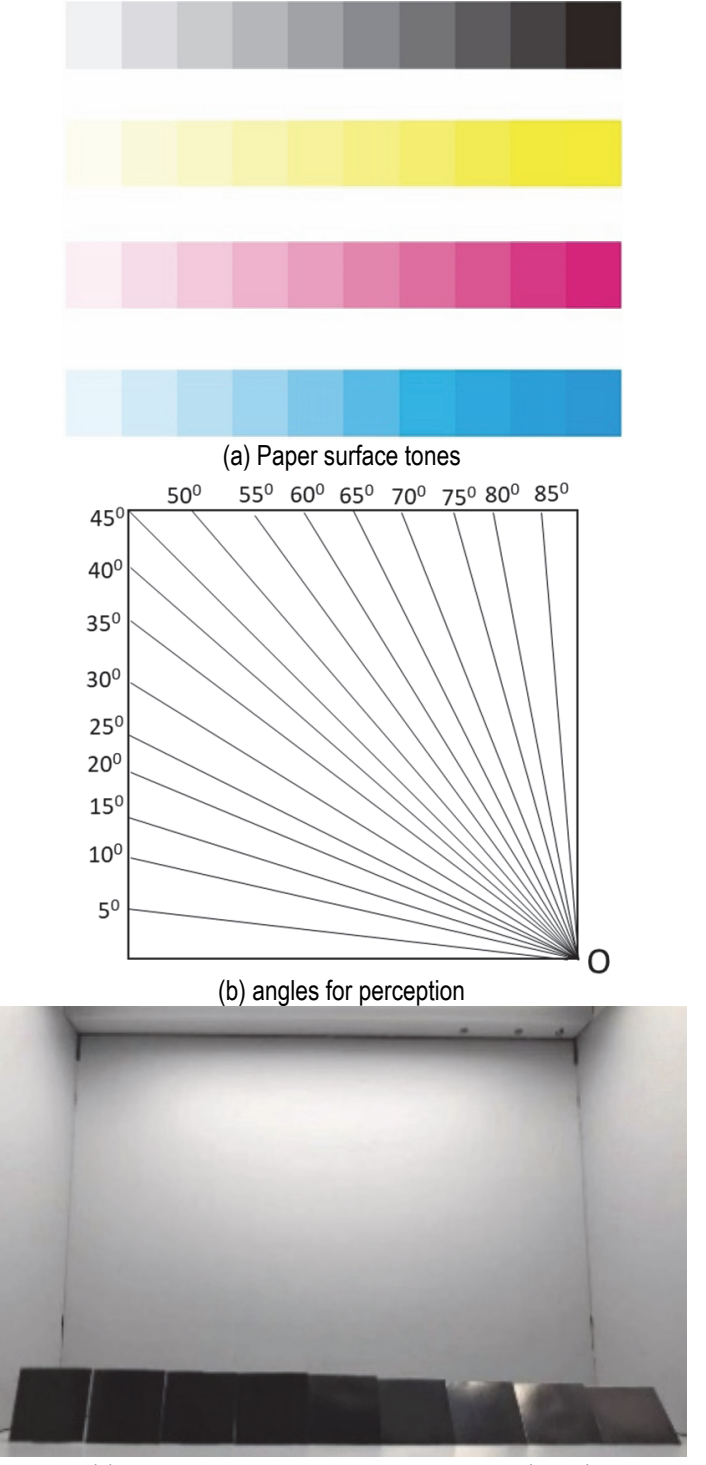

(c) rotating bracket with paper sample sets (black)

Figure 5 paper samples with inks, angle configurations and rotation equipment

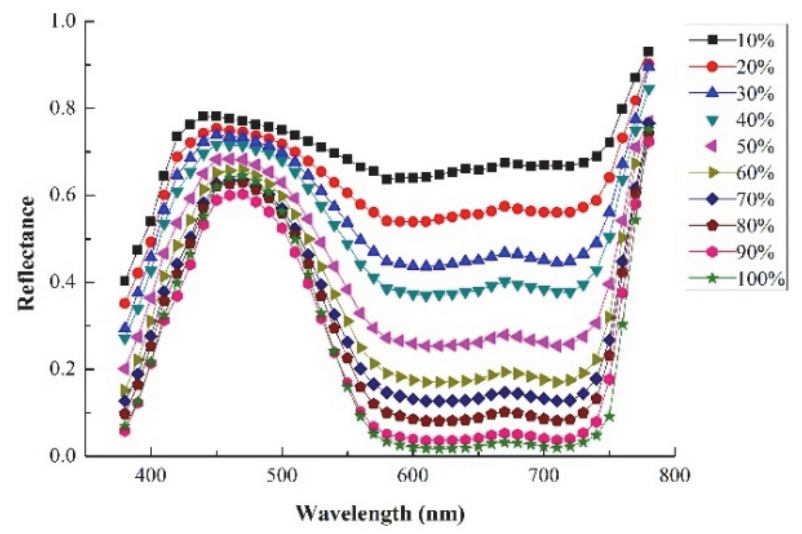

Figure 6 reflectance of paper samples in 2D plane

\subsection{Measurement design}

A spectroradiometer (Konica Minolta), CS-2000, was used to measure the spectral radiance factor of paper product surface. Several measure sensor models are also used in the experiments. The sensor position, i.e., CS2000, and light source position, standard observation environment, are fixed. And the sample position is set to be able to rotate around a bracket designed in the experiment. The configuration diagram is shown in Fig. 7.

The luminance of paper model sample is at the same conditions of illumination and geometry as the visual evaluation. A standard illumination with colour temperature of $6500 \mathrm{~K}$ was used as a light source. The measurement area and lighting area were set in a dark room under the standard observation box, Fig. 7. Here the illumination level is set as 6001x, and the luminance value was $120 \mathrm{~cd} / \mathrm{m}^{2}$ under the normal incidence and viewing situations.

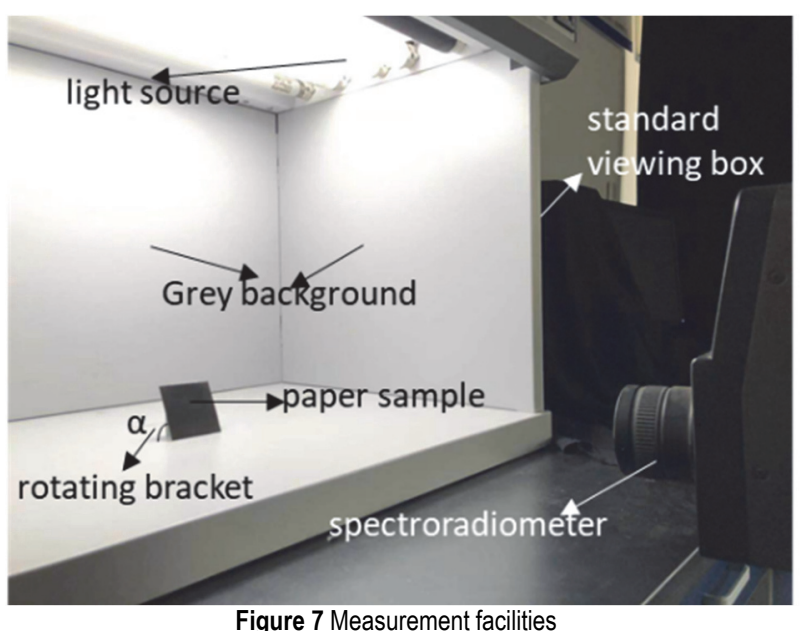

\section{RESULTS AND DISCUSSIONS}

Although the incidence angle is an important parameter in visual assessment, it should be noted that incident light is fixed in a stable position. The spectral curve is showed as a function of viewing angle. Furthermore, another term, tone, is an important factor. To classify this point clear, the tone factors were also described. The visible range and the stimulus were plotted as a function of the tones under the same perceived angle.

\subsection{Luminosity with Angle Changes}

For luminosity, the angle of $30^{\circ}$ has the maximum value, Fig. 8. The radiance intensity perceived would have a peak at the same tone. For the tone factor, the stimulus decreases obviously with the tone increasement.

For Cyan, the maximum value difference is diffmax $=$ 23.61, while it is less obvious than the minimum value difference which is diffmin $=43.59$ for every tone and viewing angles. And we can also find that the stimulus is linear to the tone change in general. The stimulus is less sensitive on $30^{\circ}$ than that on other degrees.

For Magenta, the maximum value difference is diffmax $=22.74$, while it is less obvious than the minimum value difference which is diffmin $=43.14$ for every tone and viewing angles. And it is obvious that it has the similar situation to the Cyan. The tone $40 \%$ and $90 \%$ have small differences from that in Cyan. They interact with other tone stimulus.

For Yellow, the maximum value difference is diffmax $=3.4$, while it is less obvious than the minimum value difference which is diffmin $=11.67$ for every tone and viewing angles. We also can find that the stimulus $Y$ is less 
sensitive than other tones. And it showed that the angle has more complex effect on the $Y$ stimulus.

For Black, the maximum value difference is diffmax = 22.26, while it is less obvious than the minimum value difference which is diffmin $=52.51$ for every tone and viewing angles. It is still like that in Cyan and Magenta, however at the diffmin position, it shows the smaller angle is more sensitive to perception than that in other colour tones.
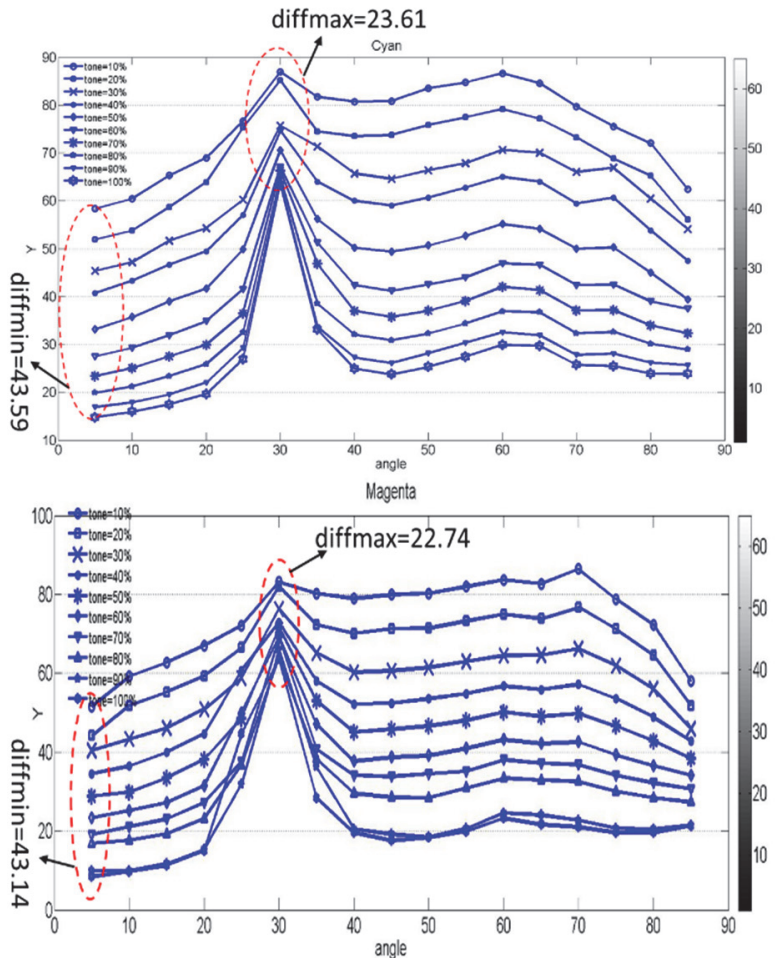

Yellow

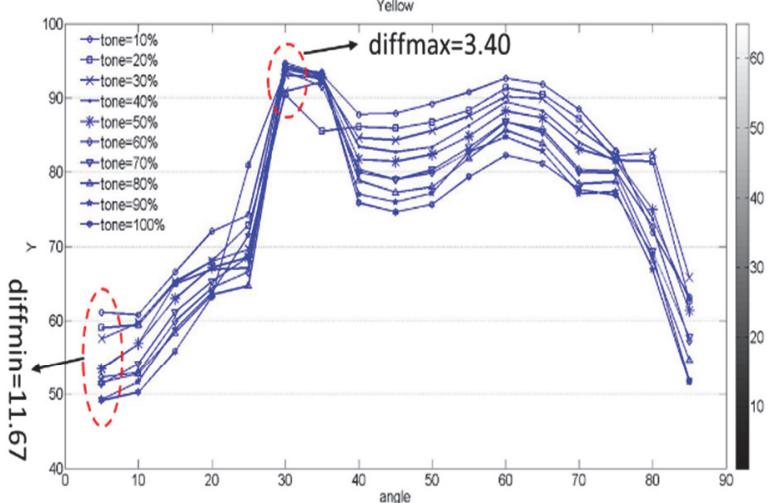

Black

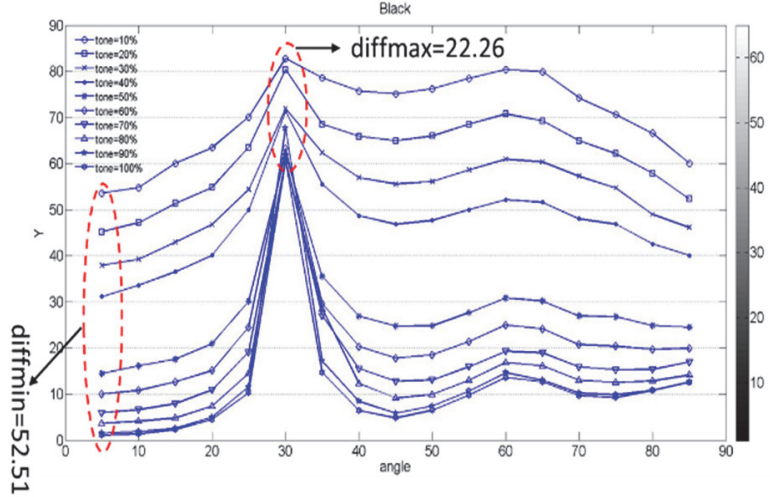

Figure 8 Luminosity with angles' changes of Cyan, Magenta, Yellow and Black

\subsection{Luminosity with Tone Changes}

For luminosity, it decreases with tone increasement obviously no matter what the perceived angles change except for Yellow tone. And the luminosity for $30^{\circ}$, it has the highest luminosity and is less sensitive to the tone change, of course not included in Yellow tone.
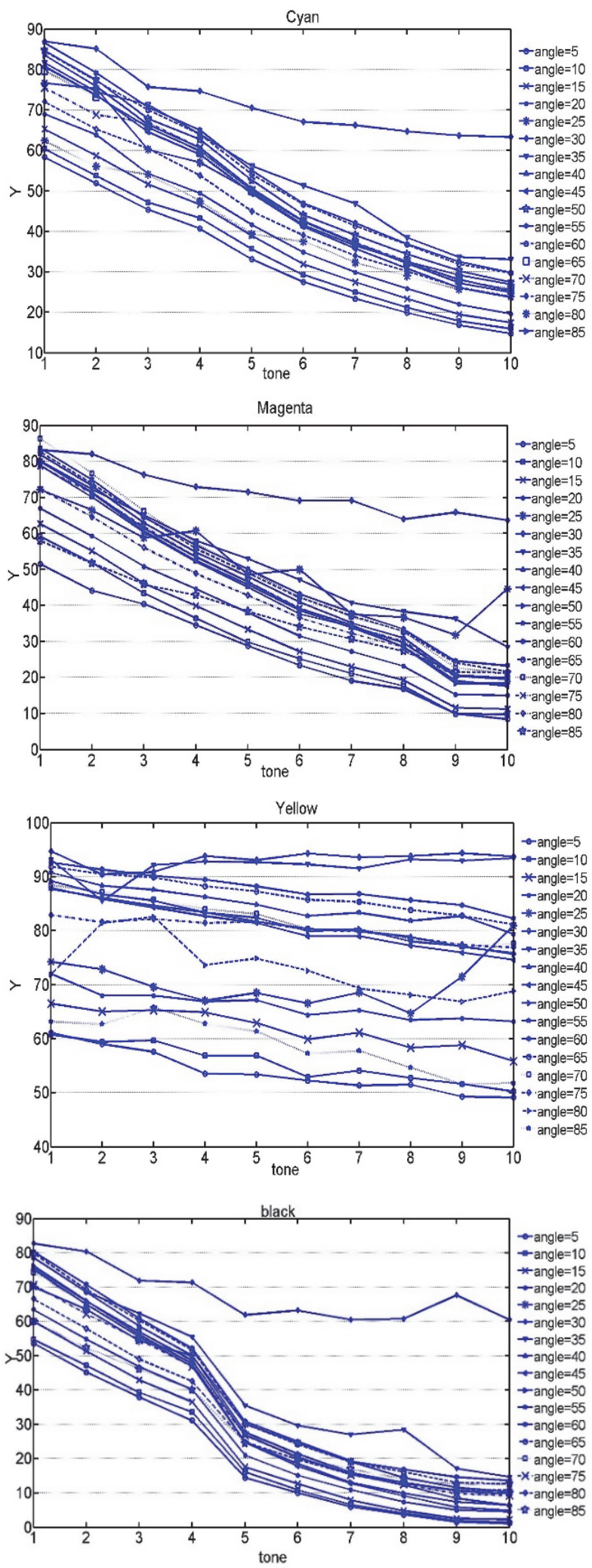

Figure 9 Luminosity with tones of Cyan, Magenta, Yellow and Black 
In Fig. 9, it is easy to find that all the tones have similar effect on stimulus under different perceiving angles except the yellow tone, though there are some differences among them. In Cyan, Magenta and Black, the angle $30^{\circ}$ has the highest stimulus values compared to other angles and is recognized easily. For Yellow tone, we found that the stimulus is very different from the other three tones. the $30^{\circ}$ is less sensitive than that in other tones. And the stimulus is obviously higher than that in other tones, e.g., the stimulus starts from 50 while others start from 10 or so. Furthermore, the stimulus changes insensitively in Yellow tone compared to other three tones.

\subsection{Luminosity with Dual Terms}
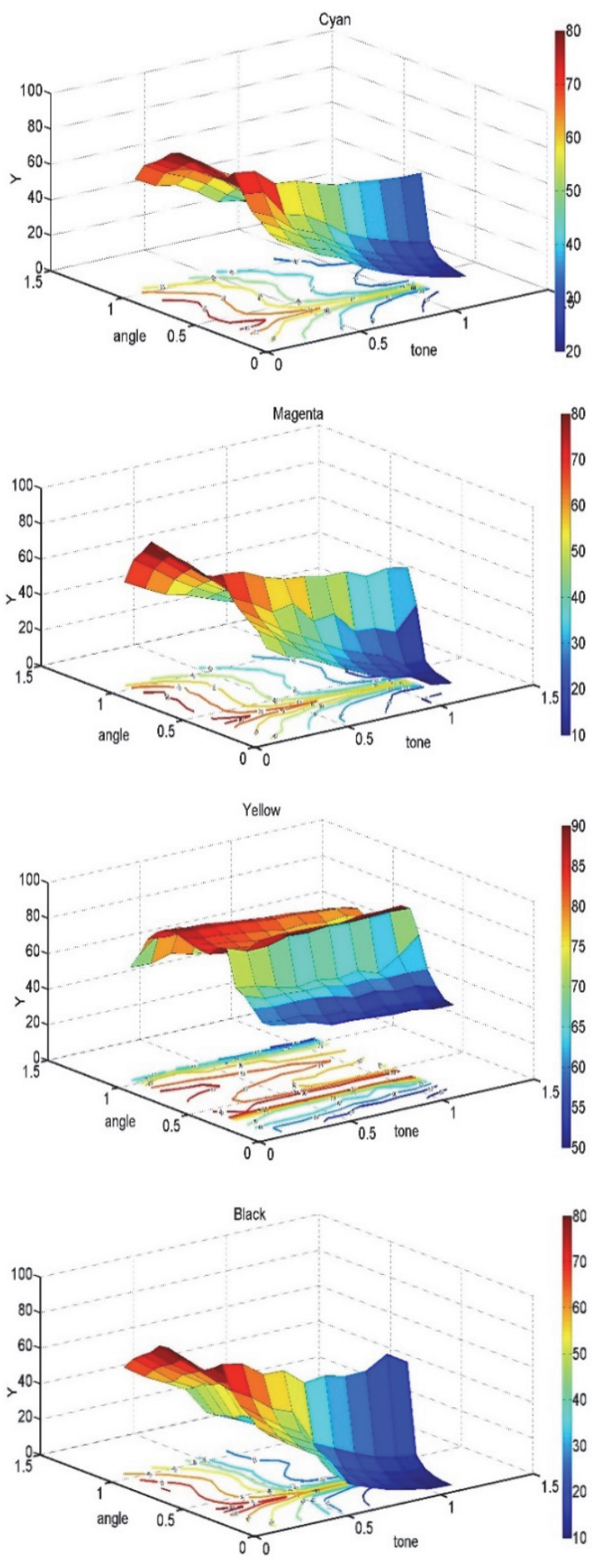

Figure 10 Luminosity with dual-factor of angle and tone
From both terms analysis, tone and angle, we found that the luminosity, or other terms used to describe the intensity of perceived lightness of the paper model surface under such configuration and viewing conditions, is affected by two main terms. It is a dual variable function which is composed of tone and angle. It is shown in Fig. 10. From the contour lines, we also can find that the relations among perceived luminosity, tone and angle are layered, segmented and nonlinear. And we will do another research to find the relations among them and try to model them in another paper.

\subsection{Perceiving Reflectance Intensity}

From the experiment, we found that no matter the angle and tone change or not, the reflectivity is also similar. So, we only take one group charts to give the analysis. Although both angle and tone are different, the reflectance has similarity to the plain paper. It is less effected by the dual terms in this paper. In Fig. 11, what we perceived is quite consistent with the measurement data. It showed that reflectivity would change less or have similar properties like that in plain paper.
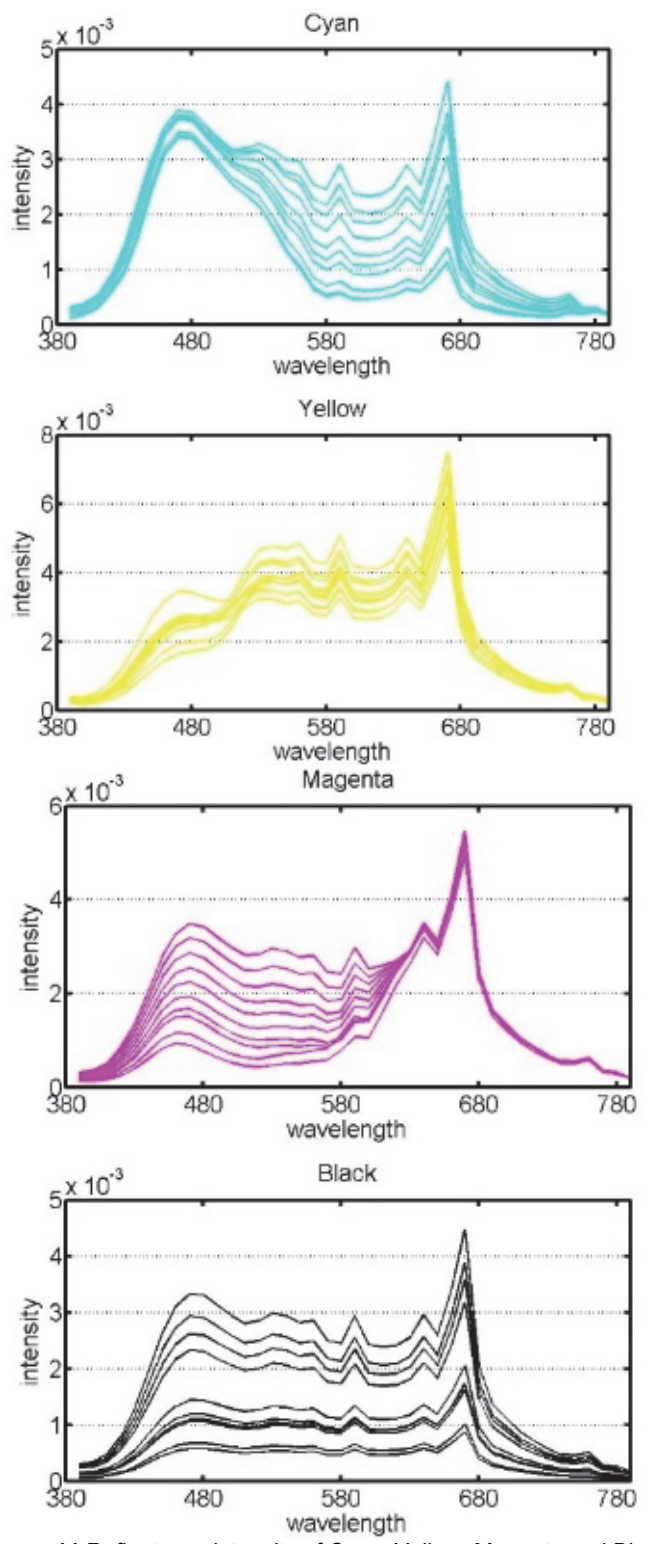

Figure 11 Reflectance intensity of Cyan, Yellow, Magenta and Black 


\subsection{Colour Coordinates in CIE1931XYZ Space}

For coordinates shown in Fig. 12, Cyan, Yellow and Black have better equilibrium distribution in colour space, except the Magenta. It shows that no matter the perceived angle or tune changes, its chroma keeps consistent in the same hue angle while its luminosity would change. And the Magenta is easily to be affected by the perceived angles and the tone varies. And Yellow is less affected by that. In fact, this is always consistent with the factual perception and other analysis.
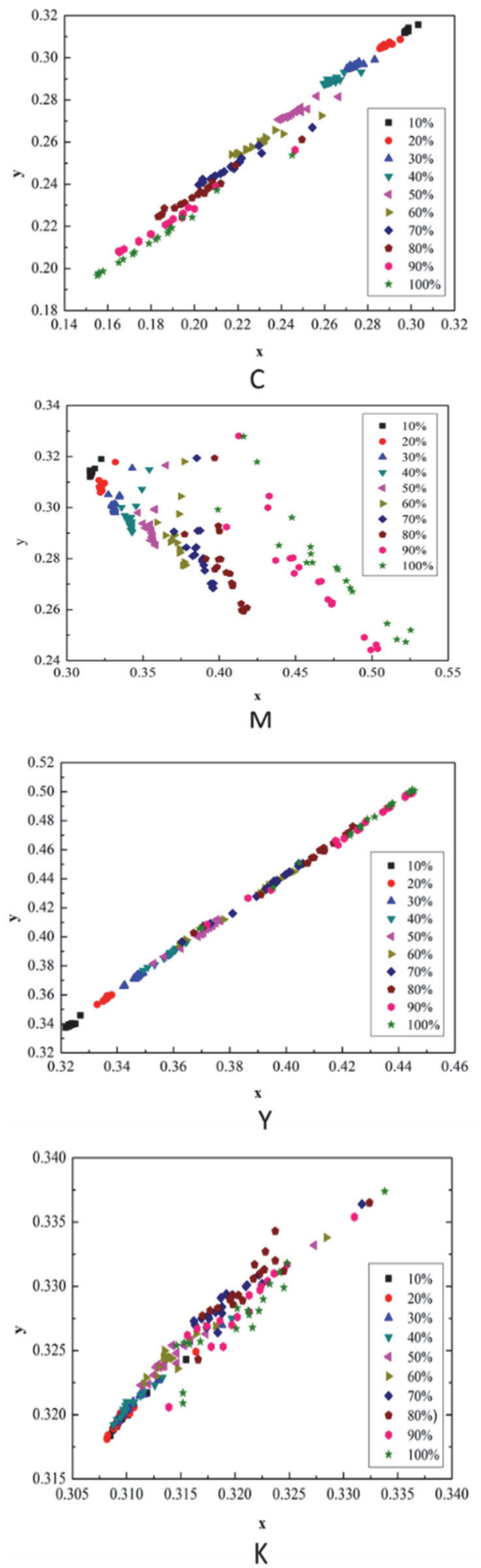

Figure 12 Coordinates of primary colours with different tones

\section{CONCLUSION}

The luminosity of paper model samples with different perceived angles and tones, was represented in this paper. Concerning the observation geometries designed in the experiment, the luminosity was perceived at the specular reflection angle and specular tone. The luminosity perception is sensitive to the perceived angle and surface tones. The colour perception of the same point on the surface will change with the perception angle. The perception of luminosity in this experiment still changes even with extreme lightness or extreme chroma. And there is a highlight point with angle of $30^{\circ}$.

It is possible to develop a mathematical model to predict the luminosity and chroma perception for paper model product by controlling the perception angles and the point tones which could predict a good colour representation on stereoscopic paper product surfaces. However, the prediction process and model are another topic in the coming research. It will be shown in next paper works.

\section{Acknowledgements}

This work was financially supported by Key Research and Development Program of Shandong Province (2019GGX105016, 2018GGX106009), State Scholarship Fund of China (201808370100), Scientific Research Foundation of Higher Education Institutions of Shandong Province (J18KA332, J17KA178), National Undergraduate Training Program on Innovation and Entrepreneurship (201810431003), Foundation of Key Laboratory of Pulp and Paper Science and Technology of Ministry of Education/Shandong Province of China (KF201604), State Key Laboratory of Pulp and Paper Engineering (201722).

\section{REFERENCES}

[1] Ikeda, N., Miyashita, K., Hikima, R., \& Tominaga, S. (2014). Reflection measurement and visual evaluation of the luminosity of skin coated with powder foundation. Color Research \& Application, 39(1), 45-56. https://doi.org/10.1002/col.21753

[2] Brunton, A., Ariskan, C. A., Tanksale, T. M., \& Urban, P. (2018). 3D printing spatially varying colour and translucency. ACM Transactions on Graphics, 37(4), 157:113. https://doi.org/10.1145/3197517.3201349

[3] Doi, M., Tanaka, N., \& Tominaga, S. (2005). Spectral reflectance estimation of human skin and its application to image rendering. Journal of Imaging Science and Technology, 49(6), 574-582.

[4] Raymond, C. (2016). Measurement for 3D printing. Retrieved from: http://www.colour.org/events/taipei/6Measurement_For_3D_Printing.pdf.

[5] Yuan, J. P., Z̄hu, M., Xu, B. H., \& Chen, G. X. (2017). Review on processes and colour quality evaluation of colour 3D printing. Rapid Prototyping Journal, 24(2), 409-415. https://doi.org/10.1108/RPJ-11-2016-0182

[6] Li, X. Z. (2012). Study on Colour Gamut Mapping Theory and Method Based on Colour Appearance Model of High Fidelity Colour Reproduction. Guangzhou, China:South China University of Technology.

[7] Tominaga, S. \& Moriuchi, Y. (2009). Principal component analysis-based reflectance analysis/synthesis of cosmetic 
foundation. Journal of Imaging Science and Technology, 53(6), 60403-1-60403-8.

https://doi.org/10.2352/J.ImagingSci.Technol.2009.53.6.060403

[8] Chen, G. X., Chen, C., Yu, Z. H., Yin, H., He, L. X., \& Yuan, J. P. (2016). Colour 3D printing: theory, method, and application. New Trends in $3 D$ Printing. https://doi.org/10.5772/63944

[9] Yuan, J. P. \& Chen, G. X. (2015). Speedup method of paperbased 3D colour printing based on slicing strategies. Applied Mechanics and Materials, 731, 269-272. https://doi.org/10.4028/www.scientific.net/AMM.731.269

[10] Sumin, D., Weyrich, T., Rittig, T., Babaei, V., Nindel, T., Wilkie, A., \& Myszkowski, K. (2019). Geometry-aware scattering compensation for 3D printing. ACM Trans. Graph., 38(4),111-1-14. https://doi.org/10.1145/3306346.3322992

[11] Nicodemus, F. E., Richmond, J. C., Hsia, J. J., Ginsberg, I. W., \& Limperis, T. (1977). Geometrical consideration and nomenclature for reflectance. National Bureau of Standards, United States. https://doi.org/10.6028/NBS.MONO.160

[12] Goesele, M., Lensch, H., Lang, J., Fuchs, C., \& Seidel, H. P. (2004). DISCO: acquisition of translucent objects. $A C M$ Transactions on Graphics, 23(3), 835-844. https://doi.org/10.1145/1015706.1015807

[13] Peers, P., Vomberge, K., Matusik, W., Ramamoorthi, R., Lawrence, J., Rusinkiewicz, S., \& Dutre, P. (2006). A compact factored representation of heterogenerous subsurface scattering. ACM Transactions on Graphics, 25(3), 746-753. https://doi.org/10.1145/1141911.1141950

[14] Tong, X., Wang, J., Lin, S., Guo, B., \& Shum, H. Y. (2005). Modeling and rendering of quasihomogeneous materials. ACM Transactions on Graphics, 24(3), 1054-1061. https://doi.org/10.1145/1073204.1073311

\section{Contact information:}

Xiaozhou LI, PhD, Associate Professor

(Corresponding author)

School of Light Industry Science and Engineering,

Qilu University of Technology (Shandong Academy of Sciences),

3501 Daxue Road, Jinan, Shandong, China, 250300

E-mail: lixiaozhou2000@163.com

\section{Mingming CUI, Ms.}

School of Light Industry Science and Engineering,

Qilu University of Technology (Shandong Academy of Sciences),

3501 Daxue Road, Jinan, Shandong, China, 250300

E-mail: 751867202@qq.com

\section{Xiaomeng HAN, Ms.}

School of Light Industry Science and Engineering,

Qilu University of Technology (Shandong Academy of Sciences),

3501 Daxue Road, Jinan, Shandong, China, 250300

E-mail: 2282839896@qq.com

\section{Guangyuan WU, PhD}

School of Light Industry Science and Engineering,

Qilu University of Technology (Shandong Academy of Sciences),

3501 Daxue Road, Jinan, Shandong, China, 250300

E-mail:wgy19882000@163.com

Jingjing LIU, Associate Professor

Shandong University of Art \& Design,

1255 Daxue Road, Jinan, Shandong, China, 250300

E-mail: foxliu919@163.com 